Original scientific paper

\title{
MANUFACTURING CAPACITY EVALUATION OF SMART JOB-SHOP BASED ON NEURAL NETWORK
}

\author{
Wang, M.\# \\ School of Information Science and Engineering, Shandong Agricultural and Engineering University, \\ Jinan 250100, China \\ E-Mail: z2013462@sdaeu.edu.cn $\left({ }^{\sharp}\right.$ Corresponding author $)$
}

\begin{abstract}
Most Chinese enterprises lack a systematic and scientific planning for intelligent manufacturing development. Therefore, this paper tries to evaluate the manufacturing capacity of smart job-shop based on improved backpropagation neural network (BPNN). Firstly, the core production factors affecting the manufacturing capacity improvement of smart job-shop were summarized, and a multi-level scale was developed for the manufacturing capacity of smart job-shop. Next, the production cost of smart jobshop was modelled, and a smart job-shop manufacturing capacity evaluation framework was established based on job-shop scheduling evaluation. After that, the sparrow search algorithm (SSA) was optimized by firefly algorithm to improve the initial weights and thresholds of traditional BPNN, and the improved BPNN was applied to evaluate the manufacturing capacity of domestic enterprises. The proposed model was proved effective through experiments.

(Received in August 2021, accepted in November 2021. This paper was with the author 2 weeks for 1 revision.)
\end{abstract}

Key Words: Smart Job-Shop, Manufacturing Capacity, Backpropagation Neural Network (BPNN), Firefly Algorithm, Sparrow Search Algorithm (SSA)

\section{INTRODUCTION}

Since the global financial crisis in 2008, manufacturers around the world have faced the plight of shrinking sales market, rising material cost, and dwindling product profit [1-6]. Many countries began to improve traditional manufacturing with information technology (IT), trying to provide personalized services, shorten delivery period, and innovate production techniques [7-14]. In recent years, China started to implement and develop intelligent manufacturing, but the works are largely exploratory [15-18]. Most enterprises remain unclear about their own development level of intelligence and automation, lack a systematic and scientific planning for intelligent manufacturing development, and fail to fully diagnose their own manufacturing capacity. Therefore, it is necessary to evaluate the manufacturing capacity of intelligent and automated job-shops of enterprises [19-22].

To optimize the allocation of manufacturing resources, Teerasoponpong and Sopadang [23] proposed a solution that eases the burden of SMEs in manufacturing capacity planning through data collection and utilization. Benedito and Corominas [24] analysed the meaning of job transfer decision in strategic capacity planning, and developed a mathematical programming model. Fang et al. [25] depicted the stochastic aggregate production planning problem as a mixed integer linear programming model, decomposed the model into four subproblems through Lagrangian relaxation, developed a heuristic program for each subprogram, and tested the programs on many instances. Peirleitner et al. [26] applied job aggregation to reduce the complexity of the simulation model, and evaluated the influence of capacity-related decisions on the shortening of setting time and the outsourcing of loads. Li [27] developed a composite linear matrix evaluation system to empirically study 32 mechanical manufacturers in a Chinese province. The results provide a realistic guide for manufacturers to improve their quality innovation capacity (QIC).

This paper relies on big data analysis to evaluate the manufacturing capacity of smart jobshop. Specifically, the core production factors of smart job-shop manufacturing capacity 
improvement were summarized, a multi-level scale was developed for smart job-shop manufacturing capacity, and smart job-shop production cost was modelled. After that, the sparrow search algorithm (SSA) was optimized by firefly algorithm to improve the initial weights and thresholds of backpropagation neural network (BPNN), and the improved BPNN was applied to evaluate the level of manufacturing capacity improvement of job-shops.

\section{MANUFACTURING CAPACITY GRADING}

As shown in Fig. 1, this paper divides the manufacturing capacity of smart job-shop to five levels. On the first level, the enterprise has substantialized the awareness of intelligent manufacturing into planning, investment, and strategic development, and gradually realized the mechanization of manufacturing, and the computerization and automation of production control. On the second level, the job-shop has basically formed a manufacturing development mechanism, and largely informatized, standardized, and visualized the manufacturing process. On the third level, the job-shop has gained certain capacity of intelligent manufacturing, and realized the digitization of manufacturing, and the integration, interaction, and sharing of production process data. On the fourth level, the job-shop has achieved lean manufacturing of jobs and collaborative operation between machines. On the fifth level, the job-shop has completed intelligent transformation of smart job-shop, and acquired the functions of intelligent analysis, prediction, and services.

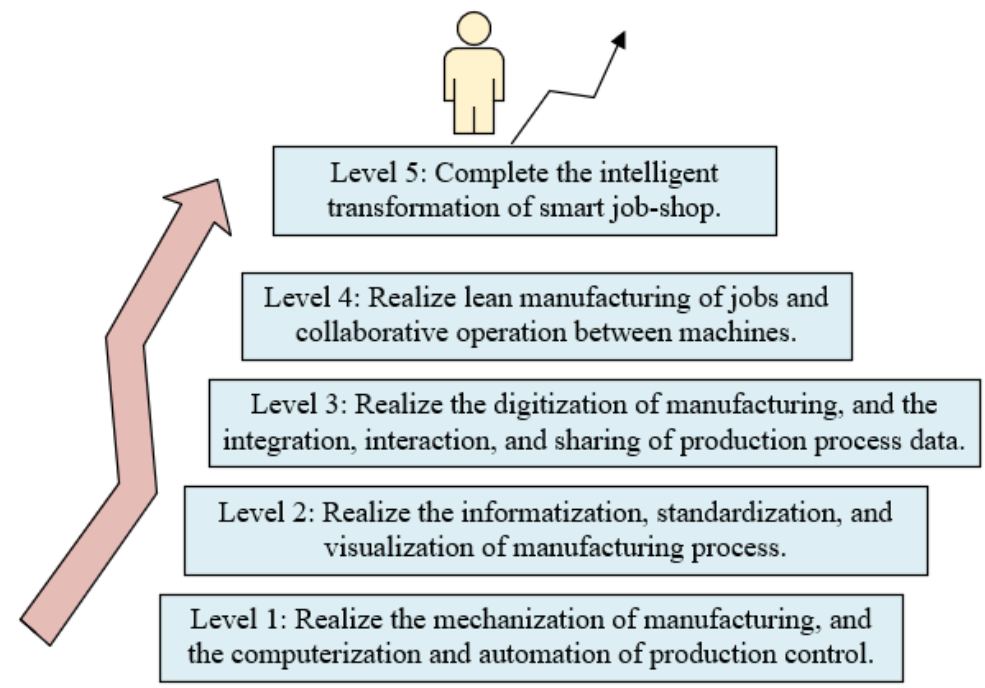

Figure 1: Manufacturing capacity levels of smart job-shop.

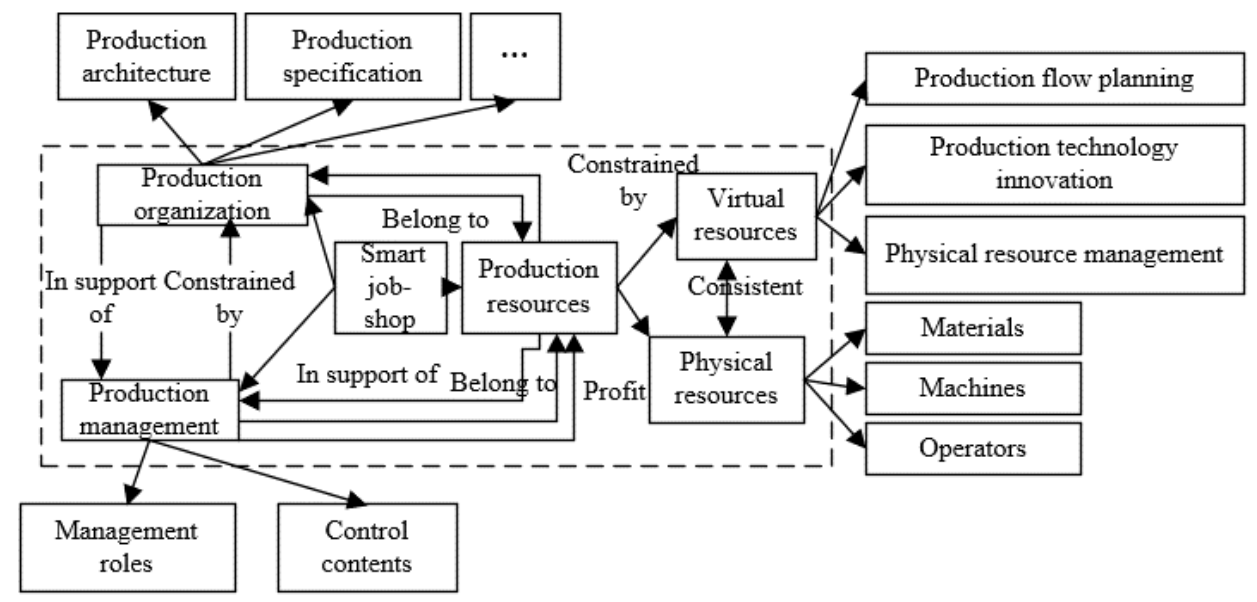

Figure 2: Architecture of smart job-shop. 
As shown in Fig. 2, the smart job-shop consists of three subsystems: production organization, production management, and production resources. Among them, production resources can be divided into two categories: physical resources like manpower, machines, and materials, and virtual resources like digital resources. In the smart job-shop, digital resources and senior technicians are more important than traditional production factors, and thus regarded as core production factors. To accurately evaluate the manufacturing capacity of smart jobshop, the evaluation model must cover six kinds of factors, including machines, logistics, inventory, digital resources, human resources, and utilities.

\section{PRODUCTION COST MODELLING}

\subsection{Production cost model}

Let $C J$ be a cost node defined to facilitate cost management; $G J$ be the connection between nodes; $O V$ be the overall cost of the production process. In the context of intelligent manufacturing, the production cost $C O$ of smart job-shop can be expressed as:

$$
C O=(C J, G J, O V)
$$

Let $X H$ be the serial number of a cost node; $L X$ be the type of a cost node; $\left\{J S_{l}\right\}$ be a cost item of the cost node. Then, the cost node $C J$ can be described as:

$$
C J=(X H, L X,\{J S\})
$$

There are six possible types $L X$ of a cost node: machine node $D N$, logistics node $L N$, inventory node $I N$, digital resource node $S N$, human resource node $H N$, and utility node $(E N)$. The cost items $\left\{J S_{l}\right\}$ mainly include depreciation, material, energy consumption, maintenance, operation, qualification, training, salary, welfare, and health.

The accounting matrix of each cost node can be established based on the attributes and accounting factors of that node. Let $L$ be the set of cost nodes; $M$ be the number of node types; $S_{l a}$ be the cost of factor $a$ of node $l ; n$ be the total number of factors. Then, the cost $J S_{l}$ of cost node $l$ can be calculated by:

$$
J S_{l}=\sum_{a=1}^{n} S_{l a}
$$

Let $C R J$ be the type of node connection, such as physical connection and information connection; $Q J$ and $H J$ be forward node and backward node, respectively. Then, the connection $G J$ between nodes can be characterized by the specific interface and topological direction:

$$
G J=(C R J, Q J, H J)
$$

The production cost model (1) of smart job-shop can be expanded hierarchically as:

$$
C O_{b}=\left(C J_{b}, G J_{b}, O V_{b}\right)
$$

Let $C O_{b}, b \in\{1,2, \ldots, H I\}$ be the production cost model of smart job-shop on level $b ; H I$ be the number of levels of the cost model, which is determined according to the levels of corporate management; $C J_{b}$ be a cost node on level $b ; G J_{b}$ be the connection between cost nodes on level $b$. From top to bottom, the cost model can be divided into the level of industrial field control $X K T$, the level of production execution and management $P X T$, and the level of production resource management GZT.

The coupling between nodes of different layers can be constructed based on the model for cost nodes on $X K T$. Further, the three cost nodes on PXT, including $H N, S N$, and $C N$ can be obtained. Referring to the correspondence between cost nodes in real production, the production cost nodes on adjacent levels are coupled. Let $C O_{c}(c=b+1)$ be the cost model of the smart jobshop on level $c ; S U\left(C O_{b}, C O_{c}\right)$ be the coupled mapping from level $b$ to level $c$. Then, the mapping between different levels can be expressed as: 


$$
\mathrm{CO}_{b} \cdot \mathrm{SU}\left(\mathrm{CO}_{b}, \mathrm{CO}_{c}\right) \rightarrow \mathrm{CO}_{c}
$$

\subsection{Job-shop scheduling evaluation}

The evaluation framework for manufacturing capacity of smart job-shop consists of three parts: job-shop production data sources, data processing and storage, and manufacturing capacity evaluation of smart job-shop (Fig. 3). Taking the intelligent manufacturing-based production cost of smart job-shop in Eq. (1) as the main evaluation index, the manufacturing capacity improvement $A I$ of smart job-shop can be defined as the gap between the current and theoretical optimal manufacturing capacities of smart job-shop:

$$
A I_{r e}=\frac{A I_{\text {tm }}-A I_{\text {best }}}{A I_{\text {best }}} \times 100 \%
$$

Let $i$ be the set of machines; $j$ be the set of tasks; $m$ be the set of event points; $r$ be the set of processes; $e \in E$ be the set of digital resources; $R_{s}$ be the set of jobs; $p=1, \ldots, P$ be the planned production cycle; $f_{r}$ be the unit inventory cost of process $r ; K C^{p}$ be the inventory of process $r$ at the end of cycle $p ; V^{p}{ }_{r}$ be the unfinished production volume of job $r$ in cycle $p ; v_{r}$ be the unit penalty cost for failing to complete the task in process $r ; s^{p}$ be the human resources invested in cycle $p ; w_{j i m}$ be the material consumed by machine $i$ to execute task $j$ at event point $m ; \omega_{j i m}$ be a binary variable reflecting whether machine $i$ executes task $j$ at event point $m ; F C$ be the fixed cost of smart job-shop. Then, the objective of the smart job-shop scheduling model can be defined as the minimization of production cost:

$$
\begin{aligned}
& \min \sum_{p} \sum_{C F_{s}} f_{r} K C_{r}^{p}+\sum_{p} \sum_{C F_{s}} v_{r} V_{r}^{p}+\sum_{p} F C_{p} s^{p}+ \\
& \sum_{p} \sum_{i} \sum_{j} \sum_{m}\left(F C_{j} \omega_{i j m}^{p}+V C_{j} w_{i j m}^{p}\right)+\sum_{p} F C_{e}^{p}
\end{aligned}
$$

Without considering the effects of utilities and logistics, the cost minimization function of smart job-shop involves inventory cost, penalty for unfinished orders in the cycle, human resource cost, machine cost, and the digital material cost of the cycle. Let $O^{p}{ }_{r}$ be the production goal of process $r$ in cycle $p ; E^{p}{ }_{r}$ be the task arranged for jobs in cycle $p$. The inventory at the end of the current cycle depends on the inventory consumption of the current cycle, the inventory increment of the current cycle, and the inventory of the previous cycle. Then, the inventories of different cycles should be constrained by:

$$
K C_{r}^{p}=K C_{r}^{p-1}+O_{r}^{p}-E_{r}^{p} \quad \forall r \in R_{S}, \forall p
$$

Let $P E_{j}$ be the machine available for task $j ; P X^{p}{ }_{r}$ be the production demand for job $r$ in cycle $p$. Since the unfinished task in the previous cycle needs to be completed in the current cycle, the cumulative unfinished task should be constrained by:

$$
V_{r}^{p}=V_{r}^{p-1}+P X_{r}^{p}-E_{r}^{p} \quad \forall r \in R_{S}, \forall p
$$

Let $N_{r m}$ be the number of event points $m$ in process $r ; C S^{p}{ }_{r}$ be the initial inventory of process $r$ in cycle $p$. During the planning for the production volume of each cycle, the actual inventory should be constrained by:

$$
N_{r, m=M}^{p}-C S_{r}^{p}=O_{r}^{p} \quad \forall r \in R_{S}, \forall p
$$

Because the initial inventory of the current cycle is the final inventory of the previous cycle, the job storage should be constrained by:

$$
C S_{r}^{p}=K C_{r}^{p-1} \quad \forall r \in R_{S}, \forall p
$$

Since a machine can only execute one task at each event point, the production process should be constrained by:

$$
\sum_{j \in J_{i}} \omega_{i j m}^{p} \leq 1 \quad \forall i \in I, \forall m \in M, \forall p
$$


Let $u^{\min }{ }_{i j}$ and $u^{\max }{ }_{i j}$ be the minimum and maximum capacities of machine $i$ in executing task $j$, respectively. The machine capacity should be constrained by:

$$
u_{i j m}^{\min } \omega_{i j m}^{p} \leq w_{i j m}^{p} \leq u_{i j m}^{\max } \omega_{i j m}^{p} \forall j \in J, \forall i \in I_{j}, \forall m \in M, \forall p
$$

Let $\psi_{S T-i j m}$ and $\psi_{E N-i j m}$ be the start time and end time of task $j$ on machine $i$ at event point $m$, respectively; $\beta_{i j}$ and $\alpha_{i j}$ be the fixed production time and yield-variable production time of task $j$ on machine $i$, respectively. Then, $\psi_{E N-i j m}$ can be obtained by superposing $\psi_{S T-i j m}, \beta_{i j}$ and $\alpha_{i j}$. The production time should be constrained by:

$$
\psi_{E N-i j m}^{p}=\psi_{S T-i j m}^{p}+\beta_{i j} \omega_{i j m}^{p}+\alpha_{i j} \omega_{i j m}^{p} \quad \forall j \in J, \forall i \in I_{j}, \forall m \in M, \forall p
$$

The end time of the current event point must precede the start time of the subsequent event point. Let $F$ be the scale variable of scheduling time. The time sequence of event points should be constrained by:

$$
\psi_{S T-i j m}^{p} \geq \psi_{E N-i j m}^{p}-F\left(1-\omega_{i j m}^{p}\right) \quad \forall j \in J, \forall i \in I_{j}, \forall m \in M, \forall p
$$

For any machine, the tasks at the current event point must be finished before the start time of the subsequent event point. The corresponding constrained can be defined as:

$$
\psi_{S T-i j(m+1)}^{p} \geq \psi_{S T-i j^{\prime} m}^{p}-F\left(1-\omega_{i j^{\prime} m}^{p}\right) \forall j, j^{\prime} \in J, \forall i \in I_{j}, \forall m \in M, \forall p
$$

The start time and end time of the current cycle must be earlier than those of the subsequent cycle. The corresponding constrained can be defined as:

$$
\begin{array}{ll}
\psi_{S T-i j(m+1)}^{p} \geq \psi_{S T-i j m}^{p} & \forall j \in J, \forall i \in I_{j}, \forall m \in M, \forall p \\
\psi_{E N-i j(m+1)}^{p} \geq \psi_{E N-i j m}^{p} & \forall j \in J, \forall i \in I_{j}, \forall m \in M, \forall p
\end{array}
$$

For any cycle, the start time and end time must fall within the same scale of scheduling time $F$. Thus, the scheduling time should be constrained by:

$$
\begin{aligned}
& \psi_{S T-i j m}^{p} \leq F \quad \forall j \in J, \forall i \in I_{j}, \forall m \in M, \forall p \\
& \psi_{E N-i j m}^{p} \leq F \quad \forall j \in J, \forall i \in I_{j}, \forall m \in M, \forall p
\end{aligned}
$$

Sufficient supply of materials is the premise of executing any task in the production process. Let $\eta_{r j}{ }^{D}$ be the proportion of material consumption $D$ of task $j$ in process $r$. Then, the production volume should be constrained by:

$$
N_{r m}^{p}=C S_{r}^{p}-\sum_{j \in J_{r}} \eta_{r j}^{D} \sum_{i \in I_{p}} w_{i j, m=1}^{p} \quad \forall r \in R_{S}, \forall p
$$

Let $N_{r}{ }^{\max }$ be the maximum material storage of process $r$. At even point $m$, the storable job volume of the smart job-shop must surpass the total job volume generated by process $r$ at that time point. The corresponding constrained can be defined as:

$$
N_{r m}^{p} \leq N_{r}^{\max } \quad \forall r \in R_{S}, \forall m \in M
$$

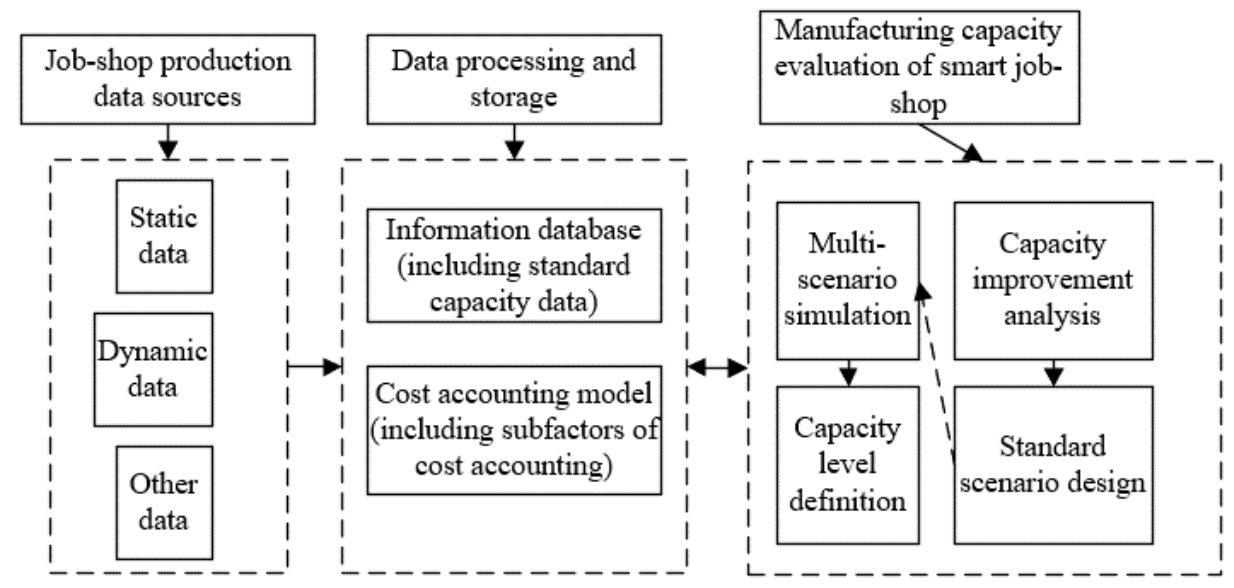

Figure 3: Evaluation framework for manufacturing capacity of smart job-shop. 


\section{EVALUATION BASED ON IMPROVED BPNN}

\subsection{Improved BPNN}

The SSA contains three behavioural strategies: finders look for food (foraging), the colony follows the finders to acquire the food (group wisdom), and the colony follows the finders to resist predation (anti-predation). In each iteration, the position of each finder is adjusted and updated. Let $\varphi$ be the current number of iterations; $A^{\varphi}{ }_{i j}$ be the position of sparrow $i$ in the $i^{\text {th }}$ dimension at the $\varphi^{\text {th }}$ iteration $(i=1,2, . ., \xi) ; Q S_{\text {max }}$ be the maximum number of iterations; $t$ be a random number in $(0,1] ; A V^{*} \in[0,1]$ be the alarm value issued by a finder during anti-predation; $A H \in[0.5,1.0]$ be the safety threshold; $T$ be a number obeying normal distribution; $K$ be an allone matrix of the size $1 \times \xi$. Then, the position of a finder can be updated by:

$$
A_{j, i}^{\phi=1}= \begin{cases}A_{j, i}^{\phi}, \exp \left(\frac{-j}{t \cdot Q S_{\max }}\right) & \text { if } A V^{*}<A H \\ A_{j, i}^{\phi}+T \cdot K & \text { if } A V^{*} \geq A H\end{cases}
$$

If $A V^{*}<A H$, there is no predator near the colony, and the finders will search for food in a wider range; otherwise, a finder notices a predator, and the other sparrows will follow the finder to quickly fly to a safe area. The hungry scroungers in the colony follow the finders to get food in various places, ready to occupy the optimal position of the finders to grab the food. Let $A_{o}$ be the optimal position occupied by a finder; $A_{w o}$ be the global worst position. Then, the position of a scrounger can be updated by:

$$
A_{i j}^{\phi+1}= \begin{cases}T, \exp \left(\frac{A_{W O}^{\phi}-A_{i j}^{\phi}}{i^{2}}\right) & \text { if } i<m / 2 \\ A_{i j}^{\phi+1}+\left|A_{i j}^{\phi}-A_{O}^{\phi+1}\right| \cdot \Phi^{+} K & \text { otherwise }\end{cases}
$$

where, $\Phi$ is a $1 \times d \xi$ matrix with elements randomly assigned as 1 or -1 . The matrix satisfies $\Phi^{+}=\Phi^{T}\left(\Phi \Phi^{T}\right)^{-1}$. If $j>m / 2$, the scrounger $j$ with a poor fitness is most likely to be hungry. Here, the proportion of finders noticing predator is set to $1 / 10-1 / 5$ of the colony. The global optimal position of finders is denoted as $A_{B E}$. Two random numbers $\gamma$ and $\delta$ are introduced to control the step length. The former is the value of normal distribution, and the latter, the flying direction of sparrows. The $\delta$ value belongs to $[-1,1]$. Let $g_{j}$ be the fitness of the current sparrow; $g_{h}$ be the best-known global fitness; $g_{q}$ be the worst-known global fitness; $\sigma$ be the minimization constant. Then, the position of the current sparrow can be updated by:

$$
A_{i j}^{\varphi+1}= \begin{cases}A_{i j}^{\varphi}+\gamma \cdot\left|A_{i j}^{\varphi}-A_{W O}^{\varphi}\right| & \text { if } g_{j}>g_{h} \\ A_{i j}^{\varphi}+\delta \cdot\left(\frac{\left|A_{i j}^{\varphi}-A_{W O}^{\varphi}\right|}{\left(g_{j}-g_{q}\right)+\sigma}\right) & \text { if } g_{j}=g_{h}\end{cases}
$$

If $g_{j}>g_{h}$, the current sparrow is on the edge of the colony; if $g_{j}=g_{h}$, a finder notices a predator, and the colony must quickly cluster to the centre position $A_{B E}$. However, the SSA has a limited global search ability, and often falls in the local optimum trap. To improve the search efficiency, the SSA was improved by firefly algorithm. Let $\lambda_{0}$ be the initial attraction of a firefly; $\mu$ be the light intensity absorption coefficient; $s_{j, i}$ be the distance between fireflies $i$ and $j$. Then, the attraction of a firefly can be calculated by:

$$
\lambda=\lambda_{0} \cdot e^{-\mu s_{j, i}^{2}}
$$

Let $a_{j}$ and $a_{i}$ be the positions of fireflies $i$ and $j$, respectively; $\beta \in[0,1]$ be a variable step size; $\mathrm{QR} \in[0,1]$ be a random disturbance. If firefly $i$ is brighter than firefly $j$, the position of the latter can be updated by:

$$
a_{j}=a_{j}+\lambda \cdot\left(a_{i}-a_{j}\right)+\beta \cdot(Q R-1 / 2)
$$


The firefly algorithm-based improvement mainly uses the firefly disturbance strategy to disturb the foraging by sparrows, aiming to optimize the position update of sparrows.

\subsection{Model flow}

As shown in Fig. 4, the initial weights and thresholds of the BPNN proposed for evaluating the manufacturing capacity of smart job-shop are improved in the following 7 steps.

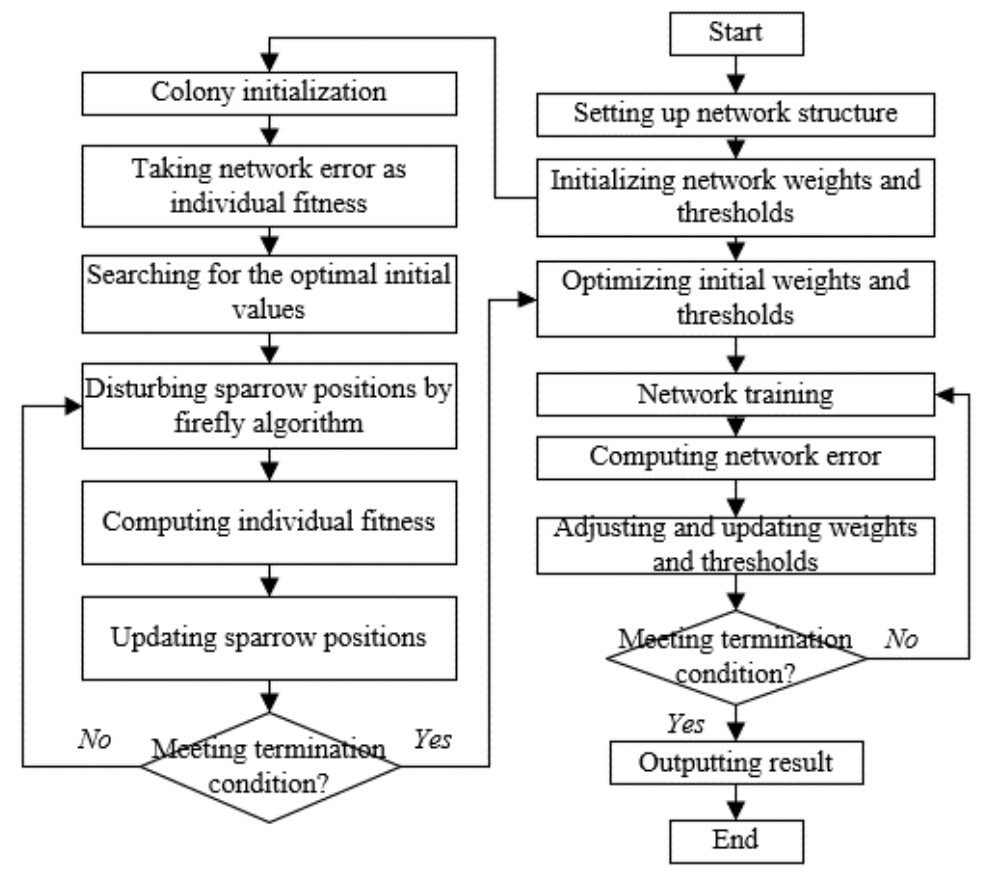

Figure 4: Flow of improved evaluation model.

Step 1. Set up the network structure.

Step 2. Initialize the colony of firefly algorithm and that of SSA. Let $S R, S C$, and $Y H$ be the number of nodes in the input layer, output layer, and hidden layer, respectively. Then, the dimensionality of the search space can be calculated based on network topology:

$$
D I M=S R \cdot Y H+S C \cdot Y H+Y H+S C
$$

Step 3. Let $m$ be the number of the evaluation samples of core production factors; $b_{j}$ be the expected output based on the samples; $b_{j}{ }^{*}$ be the actual output of the model based on the samples. The objective function of the optimized SSA can be designed as:

$$
\text { Fit }=\frac{1}{m} \sum_{j}^{m}\left(b_{j}{ }^{*}-b_{j}\right)^{2}
$$

Step 4. Calculate the new position of each sparrow according to the position update formulas for each type of sparrows in the SSA, and then derive the fitness of each sparrow.

Step 5. Disturb the foraging behaviour with firefly disturbance strategy, and iteratively search for the optimal solution with the optimized algorithm, until meeting the termination condition.

Step 6. Determine the initial weights and thresholds between different nodes, based on the matrix vector of the final optimal solution. The initial weight matrix from the input layer to the hidden layer can be expressed as:

$$
Q_{1}=a\left(1: S R^{*} Y H\right)
$$

The initial threshold matrix of the hidden layer can be expressed as:

$$
C_{j}=\left(S R^{*} Y H+1: S R^{*} Y H+Y H\right)
$$

The initial weight matrix from the hidden layer to the output layer can be expressed as: 


$$
Q_{2}=a\left(\begin{array}{l}
S R^{*} Y H+S C^{*} Y H+ \\
Y H+S C^{*} S C
\end{array}\right)
$$

The threshold matrix of the output layer can be expressed as:

$$
C_{2}=a\left(\begin{array}{l}
S R * Y H+Y H * S C+1: \\
S R * Y H+Y H+Y H * S C+S C
\end{array}\right)
$$

Step 7. Run the BPNN with the improved initial weights and thresholds, and perform error backpropagation via gradient descent until meeting the termination condition.

\section{EXPERIMENTS AND RESULTS ANALYSIS}

Fig. 5 compares the experimental result of the proposed optimized algorithm with that of the conventional SSA. It can be observed that, the proposed algorithm found the optimal feasible solution faster, and achieved better efficiency and convergence than the SSA. To obtain the proper colony size and number of iterations, this paper compares the performance between particle swarm optimization (PSO), the SSA, and our algorithm (Table I), and BPNN was selected as the neural network for all the three algorithms. After parameter configuration, the pre-processed samples of smart job-shop manufacturing were imported to our BPNN, the BPNN improved by SSA, and the BPNN improved by PSO, respectively, for training and test.

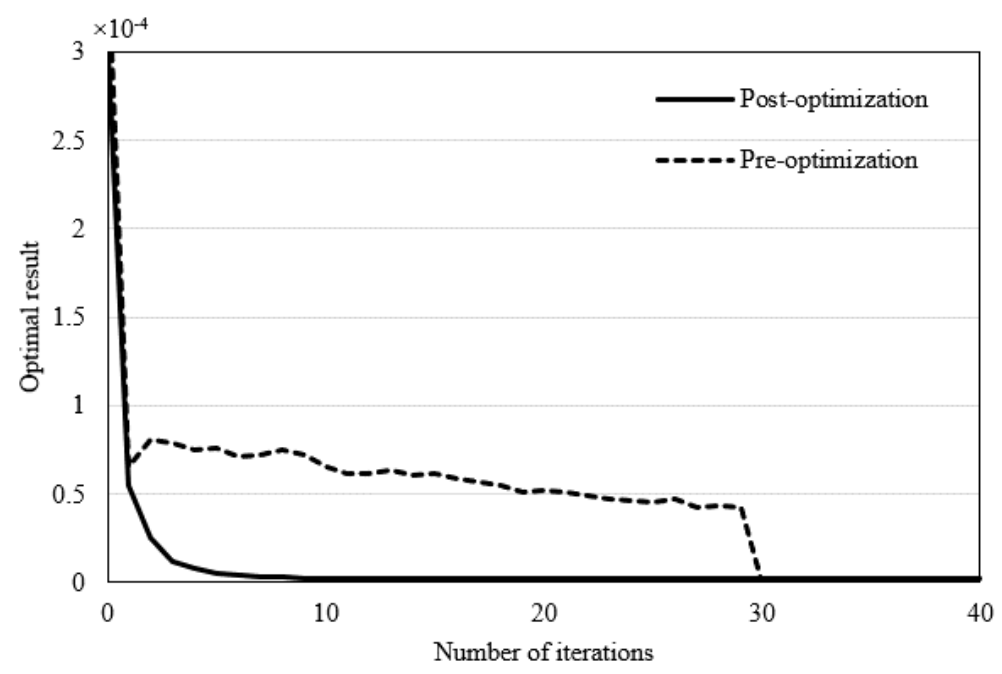

Figure 5: Experimental results on the optimized algorithm.

Table I: Parameter setting of different algorithms.

\begin{tabular}{|l|c|c|c|}
\hline \multicolumn{1}{|c|}{ Algorithm } & PSO & SSA & Our algorithm \\
\hline Colony size & 50 & 50 & 50 \\
\hline Number of iterations & 1000 & 1000 & 1000 \\
\hline Personalized parameter & 1.48752 & 0.3 & 0.1 \\
\hline
\end{tabular}

Table II: Mean values of error metrics for each model.

\begin{tabular}{|l|c|c|c|c|}
\hline \multicolumn{1}{|c|}{ Error metric } & MRE & $\boldsymbol{M S E}$ & $\boldsymbol{M A E}$ & $\boldsymbol{R M S E}$ \\
\hline Traditional BPNN & $0.4756 \%$ & $8.2415 \%$ & $25.41 \%$ & 0.2751 \\
\hline PSO-improved BPNN & $0.3254 \%$ & $5.7236 \%$ & $18.87 \%$ & 0.2534 \\
\hline SSA-improved BPNN & $0.1841 \%$ & $2.1248 \%$ & $11.54 \%$ & 0.1625 \\
\hline Our model & $0.1422 \%$ & $1.6549 \%$ & $9.751 \%$ & 0.1367 \\
\hline
\end{tabular}

Note: $M R E, M S E, M A E$, and RMSE are short for mean relative error, mean squared error, mean absolute error, and root mean square error, respectively. 


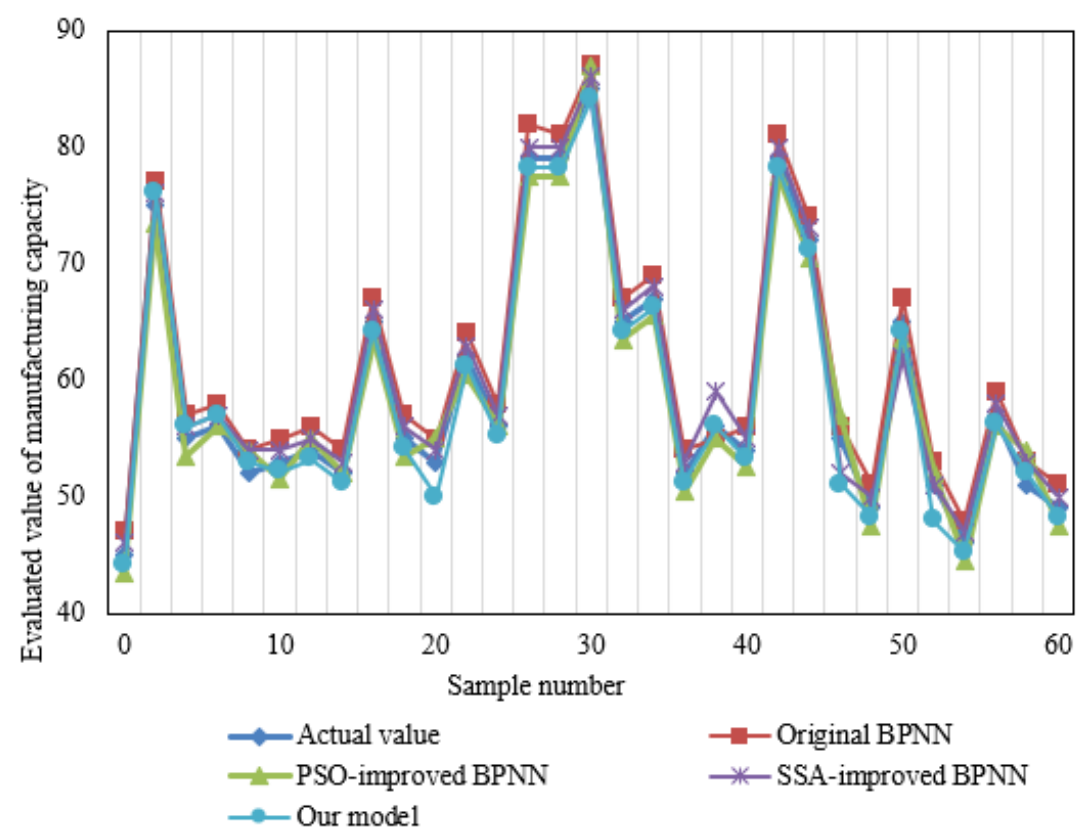

Figure 6: Evaluation results of different models vs. actual values.

The improved BPNNs may output different results in different runs. Hence, each model was run 50 times, and the mean values of its error metrics were solved (Table II). As shown in Fig. 6 and Table II, our model achieved a relatively high evaluation accuracy. This paper improves the manufacturing capacity of smart job-shop based on the core production factors. Since digital resources and senior technicians are important core production factors (Fig. 2), three simulation scenarios were selected: (1) the current manufacturing capacity scenario of the job-shop; (2) fully automated production scheme in place of semi-automated production scheme; (3) cost and scheduling-optimal control scheme in place of traditional production control scheme. Based on the simulation results (Table III), the sensitivity of each parameter was analysed under different scenarios, revealing how much the variation of production factors like $D N, L N, I N$, $S N, H N$, and $E N$ influences the model output. Table IV lists the sensitivity coefficient of each production factor, and ranks them by sensitivity.

Table III: Total costs obtained in multi-scenario simulation.

\begin{tabular}{|c|c|c|c|c|c|}
\hline Serial number & $\mathbf{1}$ & $\mathbf{2}$ & $\mathbf{3}$ & $\mathbf{4}$ & $\mathbf{5}$ \\
\hline (1)-Total cost & 375.2215 & 375.7542 & 378.9425 & 377.3452 & 378.6514 \\
\hline (2)-Total cost & 336.4251 & 316.4572 & 316.5124 & 312.5412 & 309.1246 \\
\hline (3)-Total cost & 251.9547 & 217.1542 & 217.7546 & 214.1241 & 213.5462 \\
\hline Serial number & $\mathbf{6}$ & $\mathbf{7}$ & $\mathbf{8}$ & $\mathbf{9}$ & $\mathbf{1 0}$ \\
\hline (1)-Total cost & 365.1748 & 367.5487 & 368.4781 & 365.7542 & 367.7868 \\
\hline (2)-Total cost & 274.8424 & 282.4511 & 287.2563 & 286.2245 & 289.2514 \\
\hline (3)-Total cost & 192.0913 & 199.2541 & 197.2547 & 187.2354 & 185.2786 \\
\hline
\end{tabular}

Table IV: Sensitivity coefficient and ranking of each production factor.

\begin{tabular}{|c|c|c|c|c|c|c|}
\hline Parameter & $\boldsymbol{D N}$ & $\boldsymbol{L N}$ & $\boldsymbol{I N}$ & $\boldsymbol{S N}$ & $\boldsymbol{H N}$ & $\boldsymbol{E N}$ \\
\hline Sensitivity under Scheme (1) & 123.721 & 9.425 & 5.684 & 7.368 & 115.386 & -36.059 \\
\hline Sensitivity ranking & 1 & 3 & 5 & 4 & 2 & 6 \\
\hline Sensitivity under Scheme (2) & 236.587 & -11.068 & 9.645 & 33.875 & 341.004 & -58.05 \\
\hline Sensitivity ranking & 2 & 5 & 4 & 3 & 1 & 6 \\
\hline Sensitivity under Scheme (3) & 335.725 & -14.867 & 46.564 & 17.452 & 223.087 & -62.645 \\
\hline Sensitivity ranking & 1 & 5 & 3 & 4 & 2 & 6 \\
\hline
\end{tabular}


Table V: Costs obtained in scenario-level combination simulation.

\begin{tabular}{|c|c|c|c|c|c|}
\hline Serial number & $\mathbf{1}$ & $\mathbf{2}$ & $\mathbf{3}$ & $\mathbf{4}$ & $\mathbf{5}$ \\
\hline Scenario-level combination & 5551 & 4553 & 3554 & 5531 & 5532 \\
\hline Cost & 180.4522 & 274.5875 & 310.5623 & 360.7254 & 687.8486 \\
\hline Serial number & $\mathbf{6}$ & $\mathbf{7}$ & $\mathbf{8}$ & $\mathbf{9}$ & $\mathbf{1 0}$ \\
\hline Scenario-level combination & 5433 & 5332 & 5243 & 5442 & 5244 \\
\hline Cost & 227.4526 & 255.4587 & 555.8874 & 333.5487 & 196.2658 \\
\hline Serial number & $\mathbf{1 1}$ & $\mathbf{1 2}$ & $\mathbf{1 3}$ & $\mathbf{1 4}$ & $\mathbf{1 5}$ \\
\hline Scenario-level combination & 4235 & 4331 & 4325 & 3244 & 3425 \\
\hline Cost & 273.7846 & 278.0254 & 304.2576 & 309.4526 & 354.5487 \\
\hline Serial number & $\mathbf{1 6}$ & $\mathbf{1 7}$ & $\mathbf{1 8}$ & $\mathbf{1 9}$ & $\mathbf{2 0}$ \\
\hline Scenario-level combination & 2534 & 2453 & 2432 & 2342 & 2324 \\
\hline Cost & 368.2569 & 379.5483 & 372.5624 & 386.4571 & 392.2541 \\
\hline Serial number & $\mathbf{2 1}$ & $\mathbf{2 2}$ & $\mathbf{2 3}$ & $\mathbf{2 4}$ & $\mathbf{2 5}$ \\
\hline Scenario-level combination & 2312 & 2124 & 2321 & 2214 & 1324 \\
\hline Cost & 402.3524 & 429.2145 & 458.2355 & 475.2583 & 713.2542 \\
\hline Serial number & $\mathbf{2 6}$ & $\mathbf{2 7}$ & $\mathbf{2 8}$ & $\mathbf{2 9}$ & $\mathbf{3 0}$ \\
\hline Scenario-level combination & 1321 & 1213 & 1112 & 1121 & 1111 \\
\hline Cost & 745.0085 & 761.9152 & 807.0841 & 809.1451 & 811.2144 \\
\hline
\end{tabular}

As shown in Table IV, the sensitivity coefficient of each production factor varied significantly with production control schemes. By sensitivity coefficient, the top-ranking production factors were $D N, H N$, and $S N$. The rankings of the three factors changed with the production control schemes. As the smart job-shop became more intelligent and automated, $D N$ and $L N$ were less sensitive to production cost. It can also be seen that the production cost reduction of the smart job-shop could be obviously affected by choosing production control strategies of different manufacturing capacity levels. After all adjustable production factors had been up-regulated, the production cost of scenario 1 would increase. Hence, a fixed control scheme is more sensitive than the production factors. For the smart job-shop, five levels of manufacturing capacity were defined for each of the four production control processes. The multiple combinations between scenarios and levels were simulated. The simulation results (Table V) reflect the manufacturing capacity improvement of each combination. The scenario, where all processes achieve the best (level 5) manufacturing capacity, could be adopted as the theoretically preferred scenario for production control.

\section{CONCLUSIONS}

This paper evaluates the manufacturing capacity of smart job-shop based on improved BPNN. Specifically, the authors constructed a model for the production cost of smart job-shop, and improved the initial weights and thresholds of BPNN with firefly algorithm-optimized SSA. Then, the BPNN improved by the optimized SSA was introduced to evaluate the job-shop manufacturing capacity of domestic manufacturers. Through experiments, the firefly algorithmoptimized SSA was proved feasible and effective against other algorithms. Next, the evaluated results of models improved by different algorithms were compared with the actual values, and their mean values of error metrics were calculated, revealing that our model can effectively solve the manufacturing capacity evaluation for smart job-shop. Finally, the manufacturing capacity of smart job-shop was improved based on the core production factors, and the costs were obtained through scenario-level combination simulation. The research results provide an important guide for field production control of smart job-shop. 


\section{REFERENCES}

[1] Wang, R.; Law, A.; Garcia, D.; Yang, S.; Kong, Z. (2021). Development of structured light 3Dscanner with high spatial resolution and its applications for additive manufacturing quality assurance, The International Journal of Advanced Manufacturing Technology, In review, 24 pages, doi:10.21203/rs.3.rs-409368/v1

[2] Lepore, J.; Mahmood, T.; Hartman, R. (2020). Development of a quality risk based tool for the selection of regulatory starting materials for commercial drug substance manufacturing processes, Organic Process Research \& Development, Vol. 24, No. 11, 2762-2771, doi:10.1021/ acs.oprd.0c00341

[3] Cimarosti, Z.; Bravo, F.; Stonestreet, P.; Tinazzi, F.; Vecchi, O.; Camurri, G. (2010). Application of quality by design principles to support development of a control strategy for the control of genotoxic impurities in the manufacturing process of a drug substance, Organic Process Research \& Development, Vol. 14, No. 4, 993-998, doi:10.1021/op900242x

[4] Martinez-Marquez, D.; Jokymaityte, M.; Mirnajafizadeh, A.; Carty, C. P.; Lloyd, D.; Stewart, R. A. (2019). Development of 18 quality control gates for additive manufacturing of error free patientspecific implants, Materials, Vol. 12, No. 19, Paper 3110, 31 pages, doi:10.3390/ma12193110

[5] Shen, F. (2014). The approach of improving environmental quality for the industrial interactive development of producer services and manufacture industry, Computer Modelling and New Technologies, Vol. 18, No. 12C, 1021-1025

[6] Karnieli, O.; Gur-Lavie, B. (2015). Cell therapy: early process development and optimization of the manufacturing process are critical to ensure viability of the product, quality, consistency and cost efficiency, Journal of Commercial Biotechnology, Vol. 21, No. 1, 83-91, doi:10.5912/jcb695

[7] Wechsler, J. (2015). Modern manufacturing systems key to FDA quality initiative, Pharmaceutical Technology, Vol. 39, No. 4, 16-18

[8] Huo, D. X.; Xiao, X. J.; Pan, Y. J. (2020). Multi-objective energy-saving job-shop scheduling based on improved NSGA-II, International Journal of Simulation Modelling, Vol. 19, No. 3, 494504, doi:10.2507/IJSIMM19-3-CO12

[9] Mlejnek, M.; Contreras, C.; Kim, J. (2013). P. 43: Model development for cell-gap induced Mura to improve quality of glass substrates to display manufacturers, Society for Information Display, International Symposium, Digest of Technical Papers, Vol. 44, No. 1, Book 2, 1155-1158, doi:10.1002/j.2168-0159.2013.tb06432.x

[10] Martins, E. G.; de Lima, E. P.; da Costa, S. E. G. (2015). Developing a quality management system implementation process for a medical device manufacturer, Journal of Manufacturing Technology Management, Vol. 26, No. 7, 955-979, doi:10.1108/JMTM-12-2012-0119

[11] Thomas, R. J.; Chandra, A.; Hourd, P. C.; Williams, D. J. (2008). Cell culture automation and quality engineering: a necessary partnership to develop optimized manufacturing processes for cell-based therapies, JALA: Journal of the Association for Laboratory Automation, Vol. 13, No. 3, 152-158, doi:10.1016/j.jala.2007.12.003

[12] Son, M.-S.; Yoo, K.-H.; Jang, J. (2004). Electrical simulation of the flicker in poly-Si TFT-LCD pixels for the large-area and high-quality TFT-LCD development and manufacturing, Solid-State Electronics, Vol. 48, No. 12, 2307-2313, doi:10.1016/j.sse.2004.04.008

[13] Parusov, V. V.; Starov, R. V.; Derevyanchenko, I. V.; Sychkov, A. B.; Kucherenko, O. L. (2010). A decade of quality steel production: developing a production technology for steel used in metalcord manufacture, Steel in Translation, Vol. 40, No. 1, 82-87, doi:10.3103/S0967091210010195

[14] Li, F.; Liu, W.; Bi, K. (2021). Exploring and visualizing spatial-temporal evolution of patent collaboration networks: a case of China's intelligent manufacturing equipment industry, Technology in Society, Vol. 64, Paper 101483, 18 pages, doi:10.1016/j.techsoc.2020.101483

[15] Yang, J.; Ying, L.; Gao, M. (2020). The influence of intelligent manufacturing on financial performance and innovation performance: the case of China, Enterprise Information Systems, Vol. 14, No. 6, 812-832, doi:10.1080/17517575.2020.1746407

[16] Shao, W. (2020). Impact of environmental investment on performance of intelligent manufacturing enterprises in the Yangtze river delta of China, Nature Environment and Pollution Technology, Vol. 19, No. 2, 881-888, doi:10.46488/NEPT.2020.v19i02.048 
[17] Xu, L. Z.; Xie, Q. S.; Yuan, Q. N.; Huang, H. S. (2019). An intelligent optimization algorithm for blocking flow-shop scheduling based on differential evolution, International Journal of Simulation Modelling, Vol. 18, No. 4, 678-688, doi:10.2507/IJSIMM18(4)CO16

[18] Zhou, D. D.; Xu, K.; Zhou, P.; Jiang, X. (2020). The production of large blast furnaces of China in 2018 and thoughts of intelligent manufacturing in the ironmaking process, Ironmaking \& Steelmaking, Vol. 47, No. 6, 650-654, doi:10.1080/03019233.2020.1807289

[19] Ren, J. F.; Ye, C. M.; Li, Y. (2020). A two-stage optimization algorithm for multi-objective jobshop scheduling problem considering job transport, Journal Européen des Systèmes Automatisés, Vol. 53, No. 6, 915-924, doi:10.18280/jesa.530617

[20] Jianjun, H.; Yao, Y.; Hameed, J.; Kamran, H. W.; Nawaz, M. A.; Aqdas, R.; Patwary, A. K. (2021). The role of artificial and nonartificial intelligence in the new product success with moderating role of new product innovation: a case of manufacturing companies in China, Complexity, Vol. 2021, Paper 8891298, 14 pages, doi:10.1155/2021/8891298

[21] Liu, J.; Chang, H.; Forrest, J. Y.-L.; Yang, B. (2020). Influence of artificial intelligence on technological innovation: evidence from the panel data of China's manufacturing sectors, Technological Forecasting and Social Change, Vol. 158, Paper 120142, 11 pages, doi:10.1016/ j.techfore.2020.120142

[22] Ashiquzzaman, A.; Lee, H.; Um, T.-W.; Kim, K.; Kim, H.-Y.; Kim, J. (2020). Deep learningguided production quality estimation for virtual environment-based applications, Technical Gazette, Vol. 27, No. 6, 1807-1814, doi:10.17559/TV-20200906191853

[23] Teerasoponpong, S.; Sopadang, A. (2021). A simulation-optimization approach for adaptive manufacturing capacity planning in small and medium-sized enterprises, Expert Systems with Applications, Vol. 168, Paper 114451, 13 pages, doi:10.1016/j.eswa.2020.114451

[24] Benedito, E.; Corominas, A. (2018). Embodying decisions on work shifts into strategic manufacturing capacity planning, International Journal of Production Research, Vol. 56, No. 18, 6135-6146, doi:10.1080/00207543.2017.1421325

[25] Fang, C.; Liu, X.; Pardalos, P. M.; Long, J.; Pei, J.; Zuo, C. (2017). A stochastic production planning problem in hybrid manufacturing and remanufacturing systems with resource capacity planning, Journal of Global Optimization, Vol. 68, No. 4, 851-878, doi:10.1007/s10898017-0500-6

[26] Peirleitner, A. J.; Altendorfer, K.; Felberbauer, T. (2017). Simulation based manufacturing system improvement focusing on capacity and MRP decisions - a practical case from mechanical engineering, Proceedings of the 2017 Winter Simulation Conference, 3876-3887, doi:10.1109/WSC.2017.8248098

[27] Li, W. H. (2016). Study of evaluation and empirical method on manufacturing quality innovative capacity, Key Engineering Materials, Vol. 693, 1910-1915, doi:10.4028/www.scientific.net/ $\underline{\text { KEM.693.1910 }}$ 